\title{
Versatile Implementation in Angle-Resolved Optical Microscopy: Its Application to Local Spectrometry of Microcavities with PIC-J-Aggregates
}

\author{
Yuki Obara, ${ }^{1}$ Keita Saitoh, ${ }^{1}$ Masaru Oda, ${ }^{1,2}$ and Toshiro Tani ${ }^{1,2}$ \\ ${ }^{1}$ Department of Applied Physics Graduate School of Engineering, Tokyo University of Agriculture and Technology, Naka-cho 2-24-16, \\ Kogane-i, Tokyo 184-8588, Japan \\ ${ }^{2}$ Division of Advanced Applied Physics, Institute of Engineering, Tokyo University of Agriculture and Technology, Naka-cho 2-24-16, \\ Kogane-i, Tokyo 184-8588, Japan
}

Correspondence should be addressed to Toshiro Tani, ttani@cc.tuat.ac.jp

Received 1 December 2010; Accepted 28 January 2011

Academic Editor: Kam-Sing Wong

Copyright ( $) 2011$ Yuki Obara et al. This is an open access article distributed under the Creative Commons Attribution License, which permits unrestricted use, distribution, and reproduction in any medium, provided the original work is properly cited.

\begin{abstract}
Versatile novel implementations in microspectroscopy are developed, which can provide angle-resolved optical spectroscopy at local sample areas almost in diffraction limit. By selecting focus position of light flux incident within the back focal plane of the objective lens radially from the position of the optical axis of the microscope with employing off-centered pinhole, we can obtain parallel beam with oblique incidence and its angle tuning at the sample surface. In this paper, we describe our specific optical setup and its practical working principle in detail. We report, as a demonstration of its performance, our latest studies on optical properties of cavity polariton states in the so-called quantum microcavity structures, which contain molecular Jaggregates of pseudoisocyanine (PIC) dye as active working materials. By using the microscope technique, we obtain a fair amount of improvement in the linewidth observation of cavity polariton spectra.
\end{abstract}

\section{Introduction}

With spectacular development of recent microfabrication technologies, it is increasingly important to monitor optical properties of matters at its more minute levels. As a result, the studies with microspectroscopy, the combination of traditional optical microscope and spectroscopic measurement, have been widely conducted. One of the most significant and informative spectroscopic techniques is the observation of the angle-resolved optical features. Nevertheless, implementations for such microspectroscopes have never been reported within our knowledge.

Here we have developed novel optical microscopy system which can provide observations on the angular-dependent optical features at local sample regions almost to its diffraction-limited spatial resolution. This system should possess broader utilities and work in a wide variety of situations, for example, fundamental studies such as local optical spectra of materials with specifically fabricated structures, evaluations of microfabricated photonic as well as electronic functional devices.

In this paper, specific optical microscope system with incident-angle-tunable Koehler type illumination is reported and its practical working principle as well. In addition, we introduce part of our latest studies about reflectance spectrometric measurement of molecular J-aggregates consist of pseudoisocyanine (PIC; 1, $1^{\prime}$-diethyl-2,2' -cyanine chloride) dye in metal-mirror microcavity structures, as an example of the application of such system.

The microcavities containing J-aggregates are known as the useful structures to form strong-coupling states between light and matters, the so-called cavity polariton states, even at room temperatures $[1,2]$. These hybrid quantum states have attracted much attention due not only to their fundamental physical significance [1-4] but also possible applications for low-threshold laser [5], entangled photon-pair generator [6, 7], and related future optoelectronic devices as well. 
We have been investigating, by using the planar microcavity structures, peculiar polariton features of PIC-J dispersed in polymeric thin films [2]. In the course of these studies, where the observations were basically performed in macroscopic areas with around $1 \mathrm{~mm}$ in diameters, recognitions came into us for the necessity of much higher spatial resolutions in the observations. The measurements mentioned above are inevitable to include fair amount of macroscopic inhomogeneities. As is already suggested, for example, by Agranovich et al. [8], PIC-J-aggregates dispersed in polymer thin film system should be considered as spatial and directional disordered one, and it is, therefore, rather amazing that the strong exciton-photon coupling feature appears in this system even at room temperatures. By the custom-made microspectroscopy system, we can expect first to approach some of the origins of inhomogeneities in the linewidth of cavity polariton states quantitatively, part of which will be demonstrated here.

\section{Angle-Resolved Optical Microscopy System}

Figure 1(a) shows overall optical setup schematically, which are realized by our improved total internal reflection fluorescence optics combined with ordinary inverted-type microscope (Nikon; TE-2000 U, $\times 100$ oil immersion objective lens, N.A. = 1.45). White light from tungsten halogen lamp is introduced into the microscope through specific illumination optics. Reflection images are acquired with a cooled 2D-CCD detector (Princeton Instruments; Cascade512B) placed on the imaging plane IP of the imaging lens IL. To obtain local reflectance signals, the image of the imaging lens is rotated $180^{\circ}$ to the right with further enlargement and some specified portion is sampled by $200 \mu \mathrm{m}$ core-size optical fiber OF. Spatial area on the sample surface corresponding to this core size is about $0.3 \mu \mathrm{m}$ in diameter, almost as small as the spatial resolution of the objective. The signal light through the fiber is introduced into a $300 \mathrm{~mm}$ focal-length spectrometer (Princeton Instruments; Acton SP-300i) and detected by liquid-nitrogen cooled 1D-CCD detector (Princeton Instruments; Spec-10: $100 \mathrm{~F})$.

The working principle of angler scanning illumination is described below. As shown in Figure 1(b), to get collimated incident light onto the sample surface, the beam from the lamp is once focused on the back focal plane (broken lines B in Figures 1(a) and 1(b)) of the objective, which effectively provides a point light source on the focal plane. Incident angle $\theta$ is a function of the distance from the optic axis of the microscope to the focus spot. Thus, by tuning a position of the focused spot on the back focal plane, we can scan the incident angle at the sample surface. Usually the back focal plane is located within the objective lens assembly, hence, we put the movable pinhole MP in a plane conjugate to the back focal plane, and the actual selection of specific portion of the incident light flux and the tuning of the incident angle can be provided. In case incident light flux pass through along the optical axis of the system, the incident angle is normal. Highest limit of incident angle is determined by NA of the objective lens. In our system, this limitation is around $60^{\circ}$ and the angle resolution is around $3^{\circ}$.

Next, we describe the detection system. As is shown by the optical paths (grey lines in Figure 1(c)), emitted light from one local point of the sample surface is collimated by the objective and focused by the imaging lens as imaging spot on the imaging plane IP, where the 2D-CCD detecting surface or the end of the optical fiber are situated, respectively (dashed-dot line: IP in Figures 1(a) and 1(c)). As for the incident illuminated light onto the sample, the light with incident angle $\theta$ is basically reflected with the same reflection angle $\theta$ and can be focused on the same imaging plane. Thus the reflected light from certain active sample spot, which should behave as a point source with resolution size, can again arrive at the imaging surface similarly, and be detected as real image of the sample by the 2D-CCD or part of the image by the optical fiber. Eventually, we can obtain pseudoreflection image or local spectra by the acquisition of the light within the local reflection spot (around $0.3 \mu \mathrm{m}$ ) as a part of the image.

While angle-resolved reflection measurement is demonstrated for the moment; however, it could be applicable to various type of angle-resolved microscopies as well. We expect, for example, photoluminescence, scattering and other optical measurements can be possible by rather small modifications of optical selection and tuning elements with basically the same working principle.

\section{Experiment}

In order to demonstrate microspectroscopic performance of the present system mentioned above, we provide some observation of the polariton features of quantum microcavity structures (QMSs) which contain molecular J-aggregates as optically active medium. Fabry-Perot typed planer microcavity structures are adopted here. Cavity photon energy, in this case, can be tuned by altering incident angle of illuminated light [1], where the cavity normal component is quantized. In the vicinity of resonant energy of the exciton, if its coupling strength to the photon is sufficiently large, Frenkelexciton polaritons can be generated and distinctive features in the dispersion relation will appear both in reflection and photoluminescence spectral responses of the QMSs.

The schematic diagram of the structure of our metalmirror microcavity sample is shown in Figure 2(a). The top mirror possesses sufficient transmittivity for the entering or leaving of light. The active layer is the PIC-J dispersed in PVS (polyvinyl sulfate) thin film fabricated by spincoating of their aqueous solution. The cavity length, that is, thickness of the active layer, is basically tuned to half of the wavelength $\lambda$ of the cavity photon component for light propagating through the cavity to its normal direction. The more detailed fabrication method can be obtained in [2], where the observations are all performed in macroscopic geometries.

The PIC molecules are one of the most well-known species forming J-aggregates. The chemical structure of the monomer is shown in Figure 2(b). The red (thin) and blue 

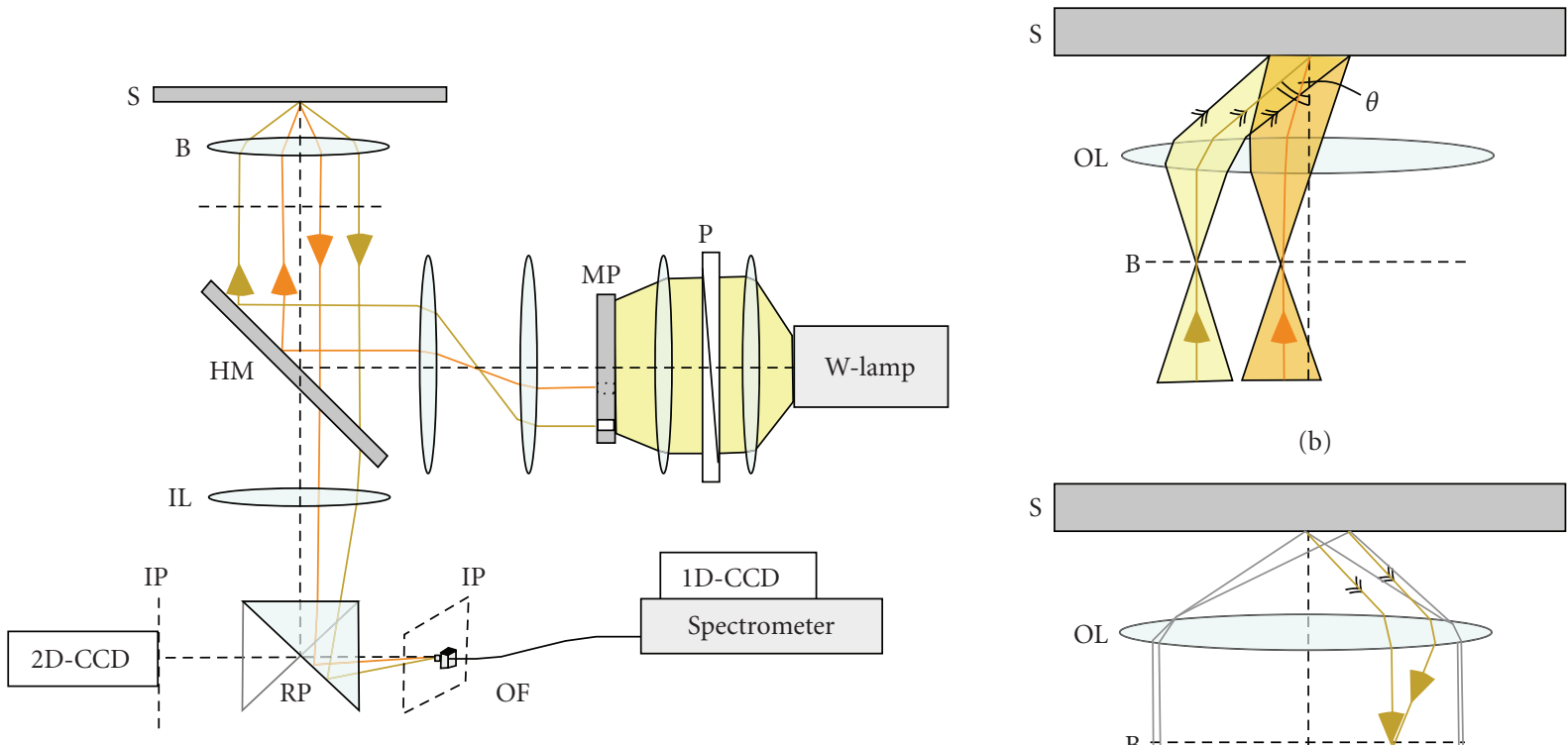

(b)

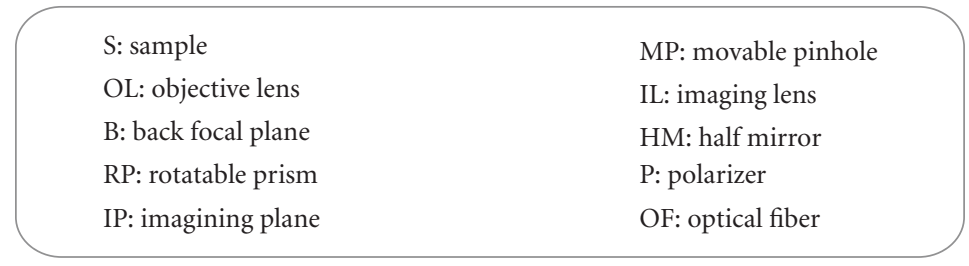

(a)

MP: movable pinhole
IL: imaging lens
HM: half mirror
P: polarizer
OF: optical fiber

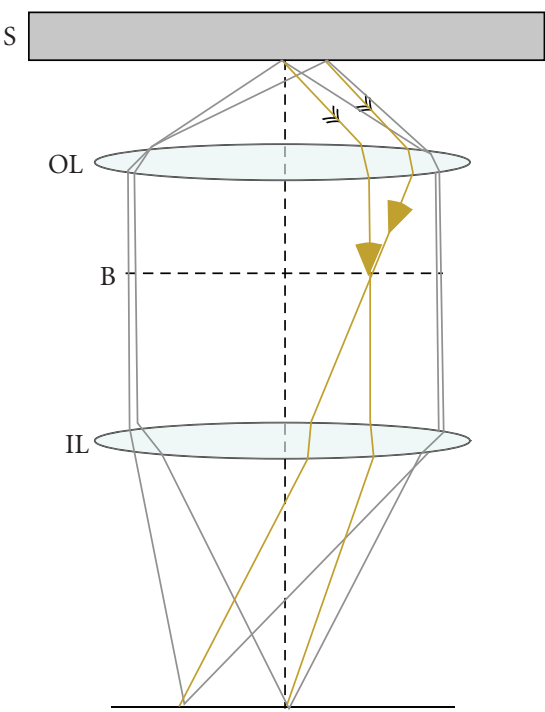

(c)

FIGURE 1: (a) Schematic microscope optics to measure angle-resolved reflectivity spectra of rather small sample area. The spatial resolution of the objective is $0.3 \mu \mathrm{m}$. Radially movable pinhole can provide effective point light source, offset from the light axis, on the aperture stop plane conjugate to the back focal plane. It eventually provides parallel beam of oblique incidence onto the sample surface. In case pinhole is on the light axis, the incident angle becomes $0^{\circ}$. If the pinhole is moved away from the center, incident angle becomes larger. Reflected light from arbitrary point of the sample surface is focused on the imaging plane, which is detected by 2D-CCD in case of imaging, and in case of local spectrometry, the image is rotated $180^{\circ}$ and specific spot is cut out by the optical fiber with $200 \mu$ m core diameter and introduced into $1 \mathrm{D}$-CCD through $300 \mathrm{~mm}$ spectrometer. Angle resolution is about $3^{\circ}$. By using polarizer, linearlypolarized incident light can also be provided. (b) and (c) show schematic illumination and imaging optics, respectively, see details in the text.

(thick) lines in Figure 2(c) show absorption and photoluminescence spectra of the so-prepared PIC-J in the aqueous solution at room temperature, respectively. The black (dotted) line shows the absorption spectrum of PIC monomers in methanol solution. A strong and narrow red-shifted band with a FWHM less than $35 \mathrm{meV}$ appears at $2.16 \mathrm{eV}$, which is attributed to the J type aggregation of the PIC molecules (J-aggregates) and is basically interpreted by Frenkel-type $1 \mathrm{D}$ exciton model. The appearance of narrow photoluminescence band with negligible Stokes shift is another prominent feature of the J-formation.

We have performed the measurement of the angleresolved reflectivity spectra of the microcavity samples by the microscopic optical system mentioned in Section 2. All experiments are executed at room temperature.

\section{Result and Discussions}

Figure 3(a) shows a typical example of microscope reflection image of the QMS sample acquired with normal incidence. Figure 3(b) shows reflectivity spectra of the samples for s-polarized incident light locally measured at the location marked by the circle with an arrow as is indicated in Figure 3(a). The two deep reflection dips are clearly seen and located at lower and upper sides of the PIC-J exciton energy (broken line). They exhibit anticrossing behavior which surely indicates the formation of cavity polaritons.

Figure 4 shows the comparison between two typical reflectivity spectra, in the vicinity of the resonant angle, which are obtained with microscopic and macroscopic optical systems, respectively. The spatial resolutions, that is, diameters of the effective local areas of the reflectance 


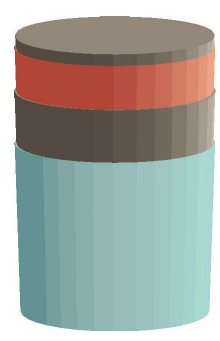

Ag $30 \mathrm{~nm}$

PIC-J/PVS $\lambda / 2$

Ag 200 nm

Silica

substrate

(a)<smiles>CCN1/C(=C/c2ccc3ccccc3[n+]2CC)C=Cc2ccccc21</smiles>

(b)

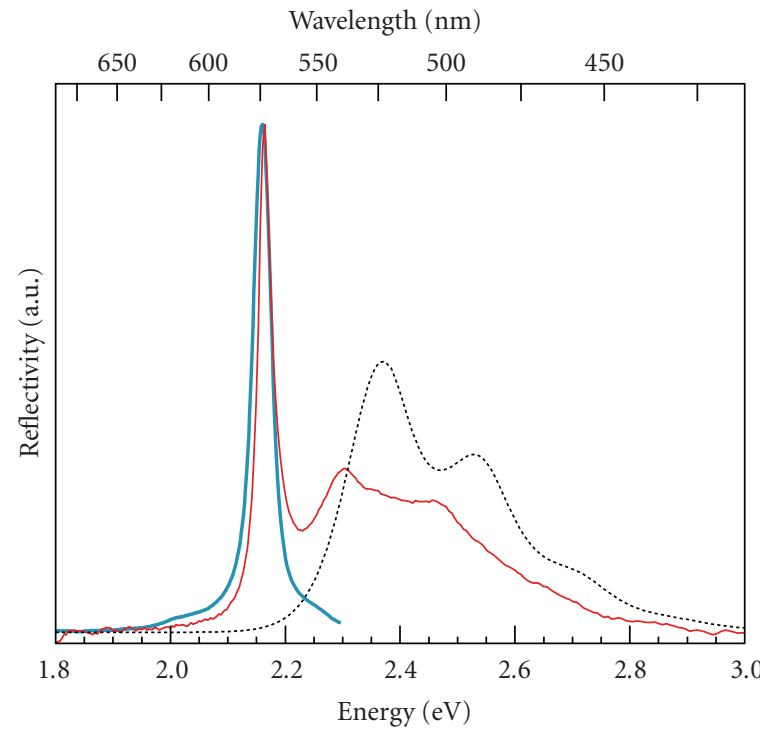

J-aggregate abs.
J-aggregate PL
$\ldots \ldots . .$. Monomer

(c)

FIgURE 2: (a) Schematic diagram of the metal-mirrors quantum microcavity structure. (b) The chemical structure of pseudoisocyanine chloride molecule. (c) Typical examples of absorption (red/thin line) and photoluminescence (blue/thick line) spectra of PIC-J-aggregates in aqueous solution of PVS polymers. Absorption spectrum of PIC monomers (black/dotted line) dispersed in methanol solution is also shown for comparison. All are measured at room temperature.

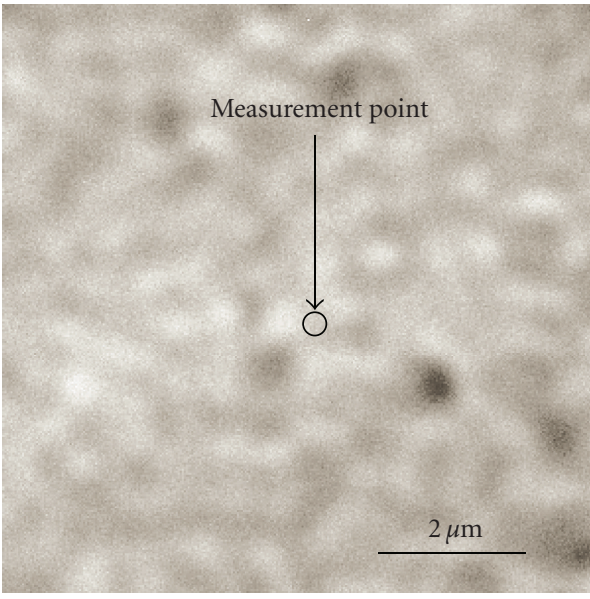

(a)

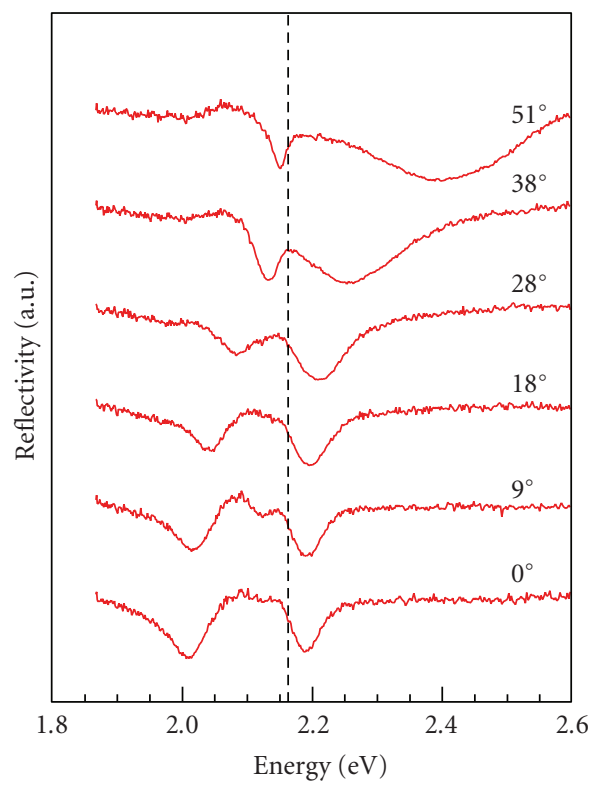

(b)

FIgURE 3: (a) Typical microscope reflection image of the microcavity sample with $8 \mu \mathrm{m} \times 8 \mu \mathrm{m}$ region under white light nonpolarized illumination. (b) Angle resolved microscope reflection spectra for the microcavity sample. Vertical broken line indicates the energy of the absorption peak of the PIC-J exciton. The incident angles for each spectrum are indicated on the right side of the figure. 


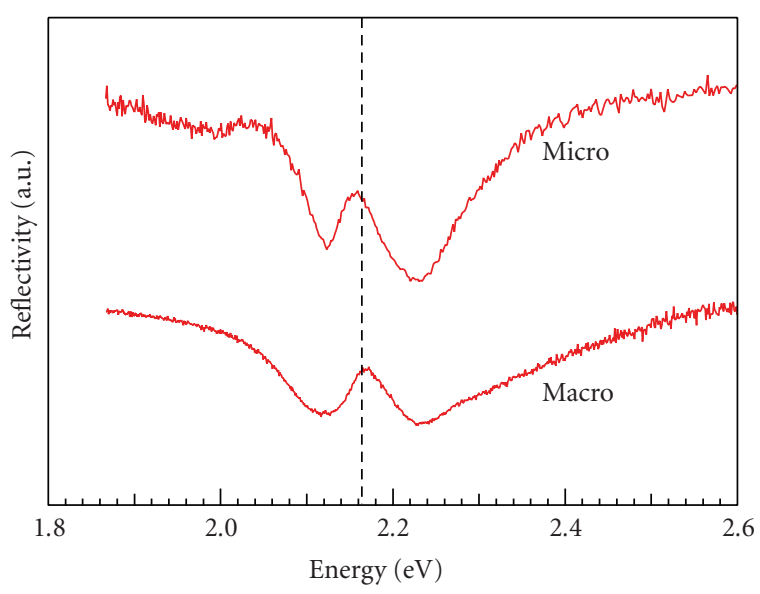

FIgURE 4: Typical reflectivity spectra measured by microscopic and macroscopic optical systems, respectively, in the vicinity of the resonant angle. The spatial resolutions are $0.3 \mu \mathrm{m}$ for microscopic and $800 \mu \mathrm{m}$ for macroscopic optical systems (see details in [2]), respectively. The vertical broken line indicates the energy of absorption peak of the J-band.

observation, are $0.3 \mu \mathrm{m}$ for microscopic and $800 \mu \mathrm{m}$ for macroscopic optical systems, respectively. As is seen in the figure, the linewidths for microscopic measurement are narrower than those for macroscopic measurement.

In accordance with the observation on almost similar cyanine dye (2,2' -dimethyl-8-phenyl-5,6,5', $6^{\prime}$-dibenzothiacarbocyanine chloride) J-aggregates system [9], the width seems basically equal to the geometric mean of the respective widths of excitons and photons near the resonant angle. Further, negligible dispersion in the electronic resonance is assumed, as is indicated by narrow linewidth of observed J-band in Figure 2(c). In our present case, the linewidth of the J-exciton is around $35 \mathrm{meV}$ and is smaller than the photon width, from $100 \mathrm{meV}$ to $130 \mathrm{meV}$ depending upon the incident angle. Hence, we consider for the moment that the observed linewidth should be mainly attributed to that of the cavity photon mode.

The linewidth of the photon mode depends on the cavity Q-factor, which is determined by the reflectivity of mirrors and energy loss of the light in the cavity [10]. The resonance frequency itself is a function of the cavity length and refractive index of active media, therefore, it can be deviated statically and spatially if the local sample thickness is inhomogeneously distributed. In our sample, distribution in the thickness of Ag mirrors is quite small and spatially homogeneous (less than 1\%). Thus, we consider that the reflectivity is almost uniform all over the sample surface. Furthermore, the distribution in the cavity length is also small, which is confirmed by the AFM thickness measurement. Remaining parameter is the effective refractive index of the active media, which can be modified with spatial inhomogeneity in the concentration of J-aggregates. We conclude, therefore, that observed narrowing of the linewidth in microscopic regime is due to the reduction of averaged inhomogeneous distribution in the J's concentration. It should, in turn, be concerned with the reduced observation area in the order of $10^{-7}$. This means that spatial characteristic length of inhomogeneous distribution of Js in PVS is in the order of $0.3 \mu \mathrm{m}$ or a bit smaller.

In addition, we can see a small dip at $2.12 \mathrm{eV}$ in Figure 3(b), especially in the spectra of incident angle of $0^{\circ}$ and $9^{\circ}$. The dip structures can be clearly confirmed only by the microscopic measurement. The existence polymorphic structures in PIC-J-aggregates is already well known and is observed just in the vicinity of the ordinary J-band [11]. Hence, we consider so far that the small dip is due to the polymorphic nature of J-aggregates. Even within the same sample, we cannot see the dips if observing points are changed. This fact also indicates that it is due to the local inhomogeneity in morphology.

Finally, we mention here briefly our two near-future strategies. First, we are now expanding J to the fibril-shaped J-aggregates: the bundle-shaped higher-order structure of Js. It is actually one of the highest concentration-limiting phases of Js [12], and the experiments on this type of aggregate phase must be informative if the microscopy is applied in the sense of QMS investigation. Secondly, time-resolved spectroscopic experiment is now under preparation, which is also quite informative if macroscopic as well as microscopic experiments are combined. These would provide further insight into the disordered nature of J-aggregate active media not only the spatial and directional disorders [8] but also various types of intra-J-aggregate inhomogeneities.

\section{Conclusions}

We have developed novel implementations in microspectroscopy system which provides angle dependent local observations of optical spectra in diffraction limit. We further demonstrate the measurement of angle-resolved local reflectivity spectra of microcavity samples containing PIC-Jaggregates. Detailed spectra are presented, whose linewidths show narrower than those of the macroscopic measurements, and are discussed briefly based on the possible inhomogeneity in microscopic distribution of the J concentration within the film.

\section{Acknowledgment}

This paper is partly supported by Grants-in-aid from the Ministry of Education, Science, Sports and Culture: nos. 22710086 and 21651066.

\section{References}

[1] R. J. Holmes and S. R. Forrest, "Coupled states of excitons, photons, and plasmons in organic structures," Organic Electronics, vol. 8, pp. 77-93, 2007.

[2] M. Oda, K. Hirata, T. Inoue, Y. Obara, T. Fujimura, and T. Tani, "Strong exciton-photon coupling and its polarization dependence in a metal-mirror microcavity with oriented PIC J-aggregates," Physica Status Solidi C, vol. 6, no. 1, pp. 288-291, 2009. 
[3] G. Panzarini, L. C. Andreani, A. Armitage et al., "Cavitypolariton dispersion and polarization splitting in single and coupled semiconductor microcavities," Physics of the Solid State, vol. 41, no. 8, pp. 1223-1238, 1999.

[4] M. S. Skolnick, T. A. Fisher, and D. M. Whittaker, "Strong coupling phenomena in quantum microcavity structures," Semiconductor Science and Technology, vol. 13, no. 7, pp. 645669, 1998.

[5] J. McKeever, A. Boca, A. D. Boozer, J. R. Buck, and H. J. Kimble, "Experimental realization of a one-atom laser in the regime of strong coupling," Nature, vol. 425, no. 6955, pp. 268-271, 2003.

[6] C. Ciuti, "Branch-entangled polariton pairs in planar microcavities and photonic wires," Physical Review B, vol. 69, no. 24, Article ID 245304, 2004.

[7] H. Ajiki and H. Ishihara, "Entangled-photon generation in biexcitonic cavity QED," Journal of the Physical Society of Japan, vol. 76, no. 5, Article ID 053401, 2007.

[8] V. M. Agranovich, M. Litinskaia, and D. G. Lidzey, "Cavity polaritons in microcavities containing disordered organic semiconductors," Physical Review B, vol. 67, no. 8, Article ID 085311, 10 pages, 2003.

[9] D. G. Lidzey, D. D. C. Bradley, T. Virgili, A. Armitage, M. S. Skolnick, and S. Walker, "Room temperature polariton emission from strongly coupled organic semiconductor microcavities," Physical Review Letters, vol. 82, no. 16, pp. 3316-3319, 1999.

[10] Y. Yamamoto, F. Tassone, and H. Cao, Semiconductor Cavity Quantum Electrodynamics, Springer, New York, NY, USA, 2000.

[11] T. Tani, L. Yi, F. Sasaki, S. Kobayashi, and H. Nakatsuka, "Dispersive line shape of persistent spectral hole-burning observed in pseudo-isocyanine bromide J-aggregates and its exciton-phonon interaction," Molecular Crystals and Liquid Crystals Science and Technology A, vol. 283, pp. 37-42, 1996.

[12] T. Tani, M. Oda, T. Hayashi, H. Ohno, and K. Hirata, "Anisotropic observation of absorption and fluorescence transition dipoles in exciton-polariton properties of PIC Jaggregates," Journal of Luminescence, vol. 122-123, no. 1-2, pp. 244-246, 2007. 


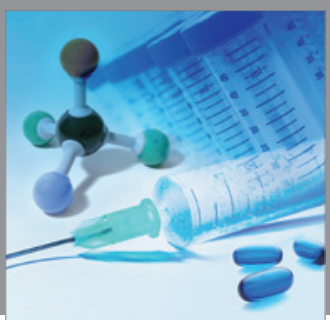

International Journal of

Medicinal Chemistry

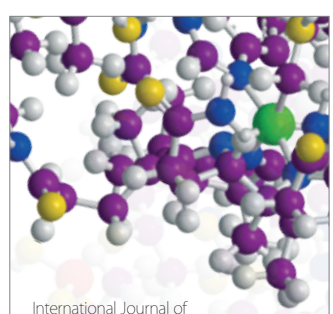

Carbohydrate Chemistry

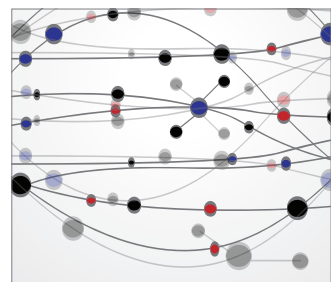

The Scientific World Journal
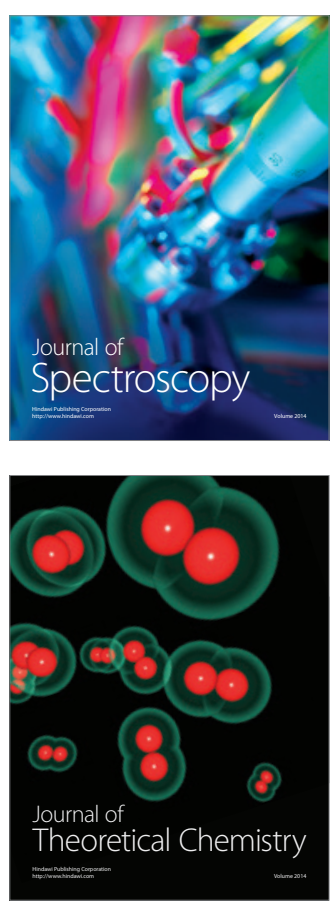
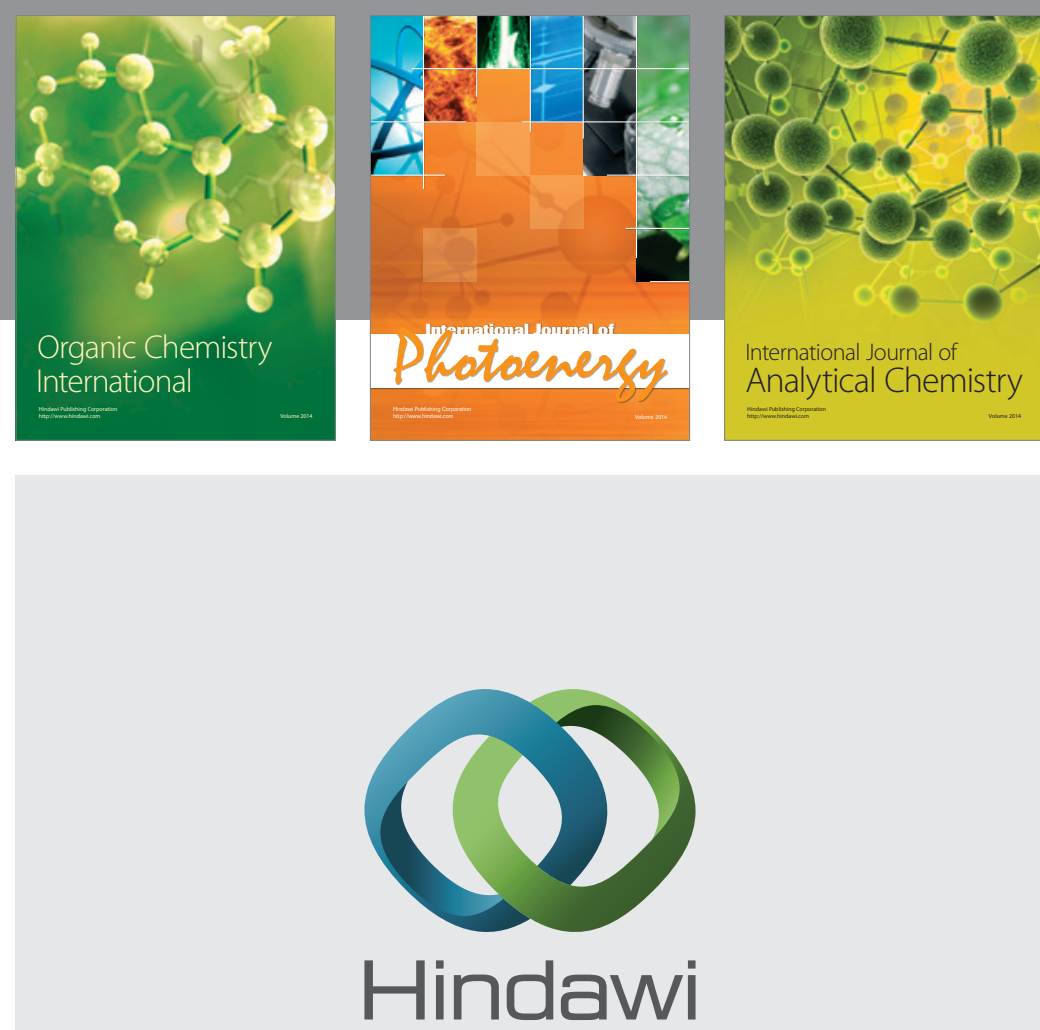

Submit your manuscripts at

http://www.hindawi.com
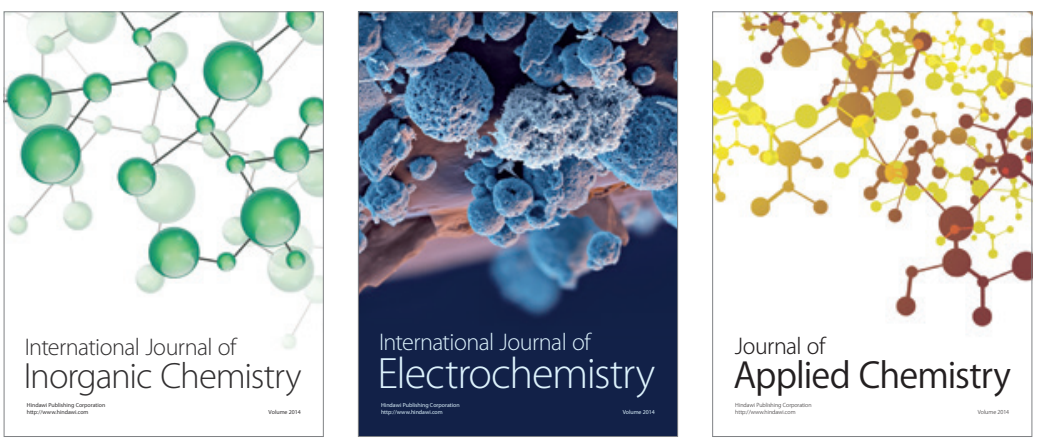

Journal of

Applied Chemistry
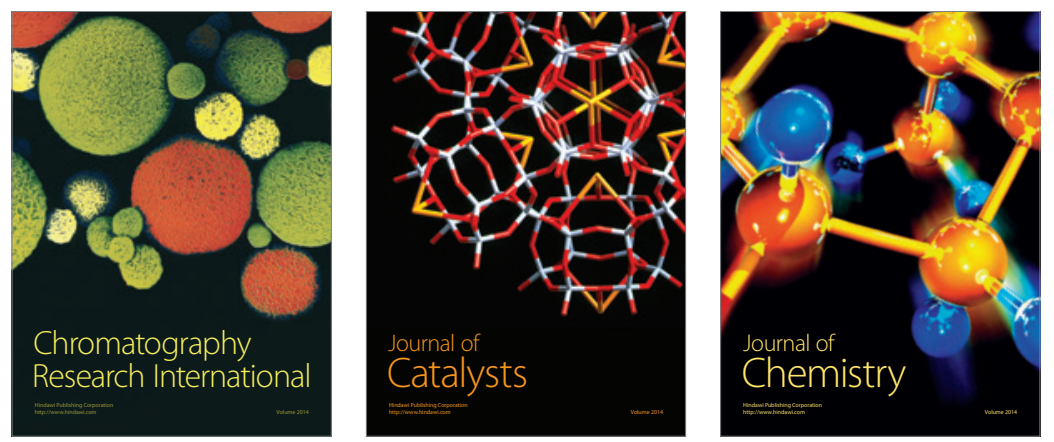
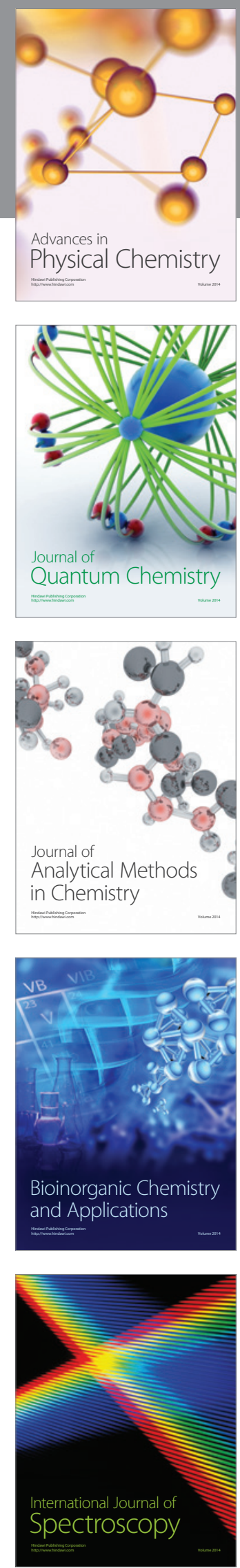